\title{
ANALISIS DE CRECIMIENTO EN Zea mays L. y Arachis hypogaea $\mathrm{L}$.
}

Julio A, Soplín *

Ana Rengifo *

Jhony Chumbe **

\section{RESUMEN}

Se realizó análisis de crecimiento en maíz (Zea mays L.) y maní (Arachis hypogaea L.) en suelo aluvial de Selva Baja de Iquitos, Perú. La máxima altura de plantas fue en maíz $282 \mathrm{~cm}$. y maní $94 \mathrm{~cm}$. El mayor número de hojas fue de $15 \mathrm{y}$ 94 respectivamente. El peso Seco por planta en maíz estuvo comprendido de 0, 11 a 217,90 g.; en maní de 0,32 a 53,50 g.

Los valores superiores encontrados de ciertos índices en maíz y maní, se expone a continuación: área foliar 169,67 y 32,11 dm² de hoja; índice de área foliar óptima 5,59 y 3,71; índice de crecimiento absoluto 79,08 y 7,68 g. $\mathrm{sem}^{-1}$; índice de crecimiento del cultivo 439,33 y $96,00 \mathrm{~g} . \mathrm{m}^{-2}$ de suelo. $\mathrm{sem}^{-1}$; índice de crecimiento relativo para ambas especies fue de $1,12 \mathrm{~g} \cdot \mathrm{g}^{-1} \cdot \mathrm{sem}^{-1}$; índice de asimilación neta 234 y 76 g.m $\mathrm{m}^{-2}$ hoja. $\mathrm{sem}^{-2}$; cociente de área foliar 310 y $198 \mathrm{~cm}^{2}$ de hoja. $\mathrm{g}^{-1}$ y duración del área foliar 1,93 y $0,44 \mathrm{~m}^{2}$ de hoja. $\mathrm{sem}^{-1}$; correspondientemente.

Se obtuvo índices de cosecha de $36 \%$ y $25 \%$ en maíz y maní respectivamente; eficiencias fotosintéticas medias de $1,27 \%$ y $0,75 \%$; además eficiencias foliares medias de 0,59 y $0,67 \mathrm{~g} . \mathrm{dm}^{-2}$ de hoja, en las especies estudiadas.

\section{INTRODUCCION}

La producción de los cultivos constituye un sistema dirigido a cosechar energía proveniente del sol, convirtiéndose en fruto, grano, forraje, fibra, aceite, madera, etc; por lo que su eficiencia debería expresarse en términos de la conversión de la radiación solar en productos finales de utilidad. Los mayores rendimientos por unidad de superficie de suelo y por año significan una conversión más eficiente de la radiación solar.

El éxito de la empresa agrícola depende del crecimiento de los cultivo. $\mathrm{Si}$ las plantas crecen y, los rendimientos de las cosechas son buenos, el agricultor obtendrá éxito con una cantidad alta en la cosecha. El crecimiento puede referirse al

* Ing. Agrónomo M. Sc. Docente Facultad Agronomía UNAP. Samanez Ocampo 185. Iquitos. Telf. 238951

** Ing. Agrónomo Jefe de Campo del Proyecto Enseñanza e Investigación GRANOS de la Fac. Agronomía UNAP 
aumento de algún órgano u órganos específicos de las plantas o a las plantas consideradas en su conjunto y pueden estar relacionadas al peso seco, longitud, altura o diámetro; pero todos ellos en función del tiempo. El modelo general es de pequeños crecimientos iniciales en tamaño, seguidos de un rápido crecimiento, después otro período durante el cual la planta crece lentamente o deja de hacerlo (curva "sigmoide"). Las curvas de crecimiento son útiles para una comprensión generalizada del proceso de desarrollo de las plantas y, mediante de esa curva determinar algunas labores culturales importantes como riego, fertilización en los cultivos, para finalmente obtener buenas cosechas.

\section{REVISION DE LITERATURA}

YOSHIDA (1972) señala que el crecimiento de biomasa en un área cultivada depende del desarrollo de su área foliar. Las hojas van cubriendo poco a poco un área disponible, aumentando gradualmente la capacidad del vegetal para aprovechar la energía solar. Además señala que un aumento en el índice de área foliar (IAF) proporciona aumento de producción de biomasa; mas, debido al autosombreamiento de las hojas, la tasa fotosintética media por unidad de área foliar decrece. Indica también el IAF ideal para determinados cultivos, Como) 3.2 para soya; 5 para maíz; de 6 a 8.8 para trigo y de 4 a 7 para arroz.

LUCCHESI (1987) menciona que la forma cónica de las plantas inducen a un mayor potencial productivo que la forma globosa; pues reduce el autosombreamiento.

MEDINA (1977) señala que para determinar el índice de crecimiento absoluto (ICA) e índice de crecimiento del cultivo (ICC), se debe realizar mediciones con intervalos de tiempo y, cuanto menores sean los intervalos, más precisas serán las mediciones del ICA e ICR. Además, como la parte productiva de la planta es la superficie foliar, es conveniente y fundamental determinar el índice de asimilación neta (IAN).

MACHADO (1985) señala que la eficiencia fotosintética (EF) de los cultivos es bajo, no alcanzando el $1 \%$ para la mayoría de las especies. La EF de un cultivo de grass produjo $51 \mathrm{~g}$. de materia seca. $\mathrm{m}^{-2}$.día ${ }^{-1}$, siendo su potencial de producción de $104 \mathrm{~g}$. de mat.seca. $\mathrm{m}^{-2} \cdot$ día $^{-1}$; pero en forma general los cultivos tienen una EF real entre 71 a $78 \mathrm{~g}$. materia seca.m ${ }^{-2} \cdot \mathrm{dí}^{-1}$.

EVANS (1983) encontró en soya que los índices de crecimiento del cultivo (ICC) durante la fase inicial oscilaban entre 8,8 y 14,9 g.m $\mathrm{m}^{-2}$.día ${ }^{-1}$ según variedad. El ICC mas alto en soya fue de 17,2 g.m $\mathrm{m}^{-2} \cdot$ día $^{-1}$ SANCHEZ (1973) encontró que los índices de cosecha (IC) en híbridos dobles de maíz amarillo duro, son bajos, así tenemos que en verano es de $23 \%$ y en invierno es de $36 \%$ en la costa peruana y de $29 \%$ para maíces de altura. 
SOPLIN (1991) encontró algunas tasas de crecimiento en dos densidades de plantación de caupí (83000 y 125000 plantas/hectáreas), así del IAF informa óptimas de 3,85 y 4,58; los valores más altos de ICC fueron de 17,17 y 20,13 g.m $\mathrm{m}^{-2} \mathrm{sem}^{-1}$; IAN de 19,70 a 125,67 y de 1,41 a 77,00 g.m $\mathrm{m}^{-2} \cdot \mathrm{sem}^{-1}$; índices de cosechas (IC) de 45 y $42 \%$.

MEDINA (1977) señala algunos índices en caña de azúcar V. 'PR- 980'; como IAN valores de 10 a $70 \mathrm{~g} . \mathrm{m}^{-2}$ de hoja. $\mathrm{sem}^{-1}$. Cociente de área foliar (CAF) valores de 20 a $60 \mathrm{~m}^{2}$ de hoja.g $\mathrm{g}^{-1}$; además ICR de 0,1 a $0,4 \mathrm{~g} \cdot \mathrm{g}^{-1} \cdot \mathrm{sem}^{-1}$; ICA de 5 a 47 g. $\mathrm{m}^{-2}$ hoja.día ${ }^{-1}$.

BENITES (1988) estimó la radiación solar en Iquitos, encontrando que la radiación global que llega a la superficie del suelo es de $16,14 \mathrm{MJ} \cdot \mathrm{m}^{-2}$.día ${ }^{-1}$ o sea $3857 \mathrm{Kcal}^{-2} \mathrm{~m}^{-2}$; es decir que la radiación que llega al suelo representa el 45,02\% del total de la radiación $\left(8568 \mathrm{Kcal} \cdot \mathrm{m}^{-2} \cdot\right.$ día $^{-1}$ - $\left.100 \%\right)$ que llega a la parte superior de la atmósfera terrestre.

\section{MATERIALES Y METODOS}

Se usó semillas de maíz (Zea rnays L.) V.'marginal 28 tropical' y maní (Arachis hypogaea L.) V.'blanco parlamento'. Los ensayos se condujeron en suelos aluviales de la "Isla Parlamento" propiedad de la Facultad de Agronomía - UNAP.

Las siembras, del maíz fue el 29.01.92 y el maní 07.02.92; usando el "puyado" con tacarpo. El maíz se distanció a 0,90 m x 0,60 m, el maní, a 0,60 m x $0,40 \mathrm{~m}$; en ambos casos se depositó 5 semillas por hoyo, para luego del entresaque dejar 3 plantas. Además señalamos que el área total de ambos ensayos fue de $900 \mathrm{~m}^{2}$ (30m x 30m) colocándose en ella los tratamientos y luego analizándolos en un diseño bloque al azar con 4 repeticiones.

El control de las malezas y de otras fitopestes fue normal, no presentándose problemas de interés durante la conducción del ensayo. En cinco plantas competitivas de ambas especies, previamente marcadas en las parcelas netas, se realizó observaciones cada diez (10) días de altura de planta, número de hojas, área foliar, peso seco total por planta y por órgano; estos datos nos permitieron encontrar diversos índices para representar gráficamente el crecimiento tal como lo propone MEDINA (1977) mediante formulas; así tenemos: índice de área foliar (IAF); índice de crecimiento absoluto (ICA); índice de asimilación neta (IAN); cociente del área foliar (CAF); duración del área foliar (DAF); eficiencia fotosintética (EF); índice de cosecha (IC); y eficiencia foliar (EF).

La cosecha de ambas, especies fue el 08.05.91, o sea a los 100 días de sembrado el maíz y, a los 90 días de sembrado el maní. 


\section{RESULTADOS Y DISCUSION}

Los cuadros 1 y 2 , consignan los datos de altura de planta y número de hojas para maíz y maní. Para el componente altura de planta, el maíz registra un rango de 16 hasta $282 \mathrm{~cm}$.; en cambio para el maní es de 11 a $94 \mathrm{~cm}$. Las curvas de crecimiento para ambas especies se presenta en los gráficos 1 y 2 , observándose la típica "sigmoide" en el crecimiento del maíz, mas no para el maní, que presenta una curva ascendente. Para el caso del maíz la depresión al final de la curva nos indica la fase final del ciclo vegetativo; en cambio para el maní la curva ascendente indica que las condiciones agroecológicas le fueron favorables para continuar con su crecimiento. El número de hojas en maíz estuvo comprendido entre 4 y 15 hojas, las que, a partir de los 60 días presentan una pequeña disminución cuantitativa de 1 y 2 unidades. Para el maní los valores estuvieron comprendidos entre 4 y 94, no observándose en ningún momento depresión en la curva, más bien constante ascenso, inclusive al momento de la cosecha. En el maíz las hojas no se incrementaron a partir de los 60 días de sembrado, mas bien disminuyeron, registrándose un envejecimiento) progresivo de las hojas, con el consecuente flujo de productos de la fotosíntesis hacia los granos. En cambio en maní, este flujo, se dio pero no hubo descenso en el numero de hojas.

Los promedios de peso seco por planta y otros órganos de maíz y maní se los presenta en los cuadros 3 y 4, respectivamente; una mejor visión de ellos se nota en los gráficos 3 y 4 . En maíz el incremento en peso seco por planta a los 20 y a los 40 días de sembrado, el ascenso es casi linear y, a partir de allí, hasta los 80 días, se registran los mayores incrementos de peso seco, para luego descender bruscamente. Para el maní se observa una típica curva "sigmoide", creciente a partir de los 20 hasta los 80 días, sufriendo ligero descenso a los 90 días, o sea a la cosecha. Respecto al incremento en peso seco del tallo, hojas, flores y frutos en ambas especies, estos tienen similitud en las curvas de peso seco total por planta. En maní se observa relación inversa entre el número de flores con el número de frutos; mientras el número de frutos es máximo a los 30 días, desciende hasta alcanzar el mínimo a los 90 días; el número de frutos es mínimo a los 40 días, alcanza el máximo al momento de la cosecha, o sea a los 90 días.

Los índices de crecimiento para ambas especies se ofrece en los cuadros $5 \mathrm{y}$ 6; mejor panorama de ellos se expone en los gráficos 5, 6, 7 y 8. En maíz la curva del área foliar (AF) es ascendente hasta los 50 días donde alcanza la mayor tasa de $169,67 \mathrm{dm}^{2}$, para iniciar un descenso progresivo; se considera que a los 50 días la planta cubrió el suelo aprovechando al máximo la radiación solar, confirmándose ello por los valores altos del índice del área foliar (IAF) encontrado de 9,43; o sea, que en un (1) metro cuadrado de suelo ocupado por el cultivo se tuvo $9,43 \mathrm{~m}^{2}$ de hojas; además que los índices de crecimiento absoluto (ICA) e índices de crecimiento del cultivo (ICC) también tuvieron los valores más altos de 79,08 g.sem1 y 439,33 
g. $\mathrm{m}^{-2} \cdot \mathrm{sem}^{-1}$ respectivamente; para estos dos últimos índices se denota a los 90 días un rápido incremento. En maní la curva del $\mathrm{AF}$ es ascendente hasta los 80 días, para luego descender a los 90 días, observándose un constante incremento del área de las hojas, suponiéndose la formación de nuevos tejidos; idéntica curva se denota para el IAF, teniendo la mayor tasa a los 80 días, siendo ella de 4,01 ó sea que en $1 \mathrm{~m}^{2}$ de suelo el cultivo tuvo $4,01 \mathrm{~m}^{2}$ de hoja; pero en estos casos los mayores valores del ICA e ICC se presentaron a los 70 días, siendo de 7,68 g. $\mathrm{sem}^{-1}$ y 96,00 g.m $\mathrm{m}^{-2}$ y 96,00 g. $\mathrm{m}^{-2}$ de hoja.sem ${ }^{-1}$ respectivamente.

El aumento del tamaño de las hojas, hasta cierto límite, en las diferentes especies produce un incremento del rendimiento; pero aclaremos que es poco ventajoso tener hojas excesivamente grandes; las hojas pequeñas son más ventajosas; además la penetración de la luz en la plantación es tanto menor cuanto más horizontal es la posición de las hojas. En casi todos los cultivos a medida que aumenta el IAF aumenta también la acumulación de materia seca, hasta cierto nivel del IAF, por encima del cual la tasa de crecimiento puede ser constante o decrecer, mientras el IAF sigue aumentando. En esta forma los mayores rendimientos son obtenidos de cultivos que tienen óptima formación de hoja.

La energía que la planta utiliza en el proceso fotosintético es interceptada por las hojas, componentes de la planta que poseen cloroplastos en sus células. En los cloroplastos se lleva a cabo la transformación de la energía solar a energía química mediante de la fotosíntesis. Los productos primarios de la fotosíntesis se transforman y se almacenan en distintos órganos de la planta en forma de carbohidratos, proteínas, etc, los cuales luego son aprovechados por el hombre.

En maíz el índice de crecimiento relativo (ICR), índice de asimilación neta (IAN), cociente de área foliar (CAF) y duración del área foliar (DAF) se presenta las observaciones en el cuadro $5 \mathrm{y}$, se muestra las curvas en el gráfico 7 , los valores para el ICR van de 0,04 a $1,12 \mathrm{~g} . \mathrm{g}^{-1} \cdot \mathrm{sem}^{-1}$; para IAN de 234,00 a $8,70 \mathrm{~g} \cdot \mathrm{m}^{-2} \cdot \mathrm{sem}^{-1}$; para CAF de 310 a $30 \mathrm{~cm}^{2} \cdot \mathrm{g}^{-1}$ y para DAF de 0,002 a $1,93 \mathrm{~m}^{2} \cdot \mathrm{sem}^{-1}$. Mientras que en el IAN se nota una curva descendente, con ligero incremento a los 90 días, siendo ello similar al ICR; en cambio para el CAF es decreciente; para DAF se nota un crecimiento ascendente con los mayores valores a los 50 y 60 días, y concuerdan con los mayores valores del IAF, en cuya etapa de crecimiento se observo la emergencia de las floraciones masculinas y femeninas. En el cultivo del maní los mismos índices se consigna en el cuadros 6 y las curvas se expone en el gráfico 8; para ICR los valores van desde 0,01 a $1,12 \mathrm{~g} \cdot \mathrm{g}^{-1} \cdot \mathrm{sem}^{-1}$, para el IAN de 76,00 a 1,59 g.m $\mathrm{m}^{-2} \cdot \mathrm{sem}^{-1}$, para el CAF de 198 a $55 \mathrm{~cm}^{2} \cdot \mathrm{g}^{-1}$ y para DAF de 0,003 a $0,44 \mathrm{~m}^{2} \cdot \mathrm{g}^{-1}$; las curvas son similares a los presendos en el maíz para los tres primeros índices; en cambio para la DAF se presenta de forma ascendente casi linear a partir de los 20 días hasta el momento de la cosecha, indicándonos la constante actividad de formación de estructuras foliares y su conservación adheridas en la planta, teniendo baja abscisión 
de las mismas lo que hace posible el constante incremento del valor de DAF en maní.

El progreso en el crecimiento del área de la hoja es determinada por el balance de formación y expansión de hojas individuales, por su longevidad y muerte $\mathrm{y}$, por el efecto de las condiciones ambientales. Al aumentar la densidad de siembra de los cultivos se logra incrementar IAF pero disminuir IAN de modo que el rendimiento llega al máximo cuando IAF logra el máximo de hojas, tan rápido como sea posible. El sombreado mutuo de las hojas no debe ser tan severo porque algunas hojas pueden volverse "parásitas" sobre la planta. El CAF expresa en cada momento la proporción del área foliar cuya fotosíntesis mantiene a toda la planta, pero relacionado con el peso seco total en un determinado tiempo. El índice DAF se refiere a la duración del funcionamiento de la superficie asimilatoria, y es importante para interpretar el costo energético de la formación de la unidad de superficie foliar de la planta y su rendimiento en la producción de asimilados.

En condiciones de campo el curso diario de la fotosíntesis muestra un periodo de máxima actividad en la mañana seguida de una caída al mediodía y un segundo máximo en la tarde. La caída al mediodía es más marcada cuando el abastecimiento de agua es inadecuado y puede estar ausente en condiciones de adecuada humedad del suelo, bajas temperaturas y presencia de nubes. La fotosíntesis neta o efectiva es variable con la especie y las condiciones ambientales; las cuales influyen sobre la pérdida diaria por respiración; para determinar exceso en la ganancia por fotosíntesis. En el cultivo del maíz la eficiencia fotosintética (EF) media fue de $1,27 \%$ o sea $5,93 \mathrm{~g} . \mathrm{m}^{-2}$. día ${ }^{-1}$, en cambio los valores de EF mínima fue de $0,02 \%\left(0,005\right.$ g.m . día $^{-1}$ al os diez días $)$, la EF máxima de 2,61\% (12,11 g.m ${ }^{-2}$ día $^{-1}$ a los 80 días). Para el maní la EF inedia fue de $0,75 \%$ lo que representa valor de 3,46 g.m $\mathrm{m}^{-2}$.día ${ }^{-1}$, en cambio la EF mínima de $0,01 \%\left(0,05 \mathrm{~g} \cdot \mathrm{m}^{-2} \cdot \mathrm{dí}^{-1}\right.$ a $\operatorname{los} 90$ días). Es necesario indicar que a estos valores debemos adicionarles en promedio el $33 \%$ del peso de los productos fotosintéticos que constituyen el desgaste realizado por la respiración, por consiguiente la fotosíntesis total o bruta son tasas mayores a las señaladas. Los valore porcentuales son bajos porque la mayoría de los cultivos cubre el suelo lentamente e intercepta una proporción considerable de radiación incidente durante el crecimiento del cultivo, siendo menor la captación de la radiación en las primeras etapas; pero señalamos que en pasturas bien desarrolladas, la captación es más eficiente que en comunidades de otras plantas. Los valores medios de EF en ambas especies estudiadas están dentro de rangos normales; porque el maíz es una planta con ruta metabólica del $\mathrm{CO}_{2}$. altamente eficiente $(\mathrm{C}-4)$ y, el maní con vía metabólica del $\mathrm{CO}_{2}$ de C-3 (SOPPLIN, 1991).

El índice de cosecha (IC) en maíz fue de $36 \%$ y en maní para fruto completo (grano + vaina) fue de $44 \%$, para grano fue de $25 \%$. Estas tasas de 
cosechas son aceptables porque los rendimientos de producto cosechable para maíz fue de 2,05 tm/ha y para maní de 1,36 tm/ha.

En relación a la eficiencia foliar (Et) en maíz, la media fue de $0,59 \mathrm{~g} \cdot \mathrm{dm}^{-2}$; la Ef mínima 0,09 g.dm ${ }^{-2}$ a los 70 días; para maní la Ef media fue de 0,67 g.dm ${ }^{-2}$, Ef mínima 0,005 g.dm ${ }^{-2}$ a los 30 días y Ef media fue de $0,67 \mathrm{~g} \cdot \mathrm{dm}^{-2}$ a los 80 días. Es interesante mencionar que la longevidad de las hojas tiene un efecto bien marcado en el rendimiento, por lo que señalamos que cuando las plantas tienen hojas longevas en actividad por varias semanas los rendimientos en las cosechas se incrementan.

\section{Cuadro 1}

ALTURA DE PLANTA (AP) Y NÚMERO DE HOJAS (NH) EN MAÍZ

CARACT.

DIAS DES PUES DE LA SIEMBRA

$\begin{array}{llllllllll}\text { EVALUAD. } 10 & 20 & 30 & 40 & 50 & 60 & 70 & 80 & 90 & 100\end{array}$

$\begin{array}{lllllllllll}\text { A.P. } & 16 & 65 & 139 & 153 & 260 & 282 & 270 & 236 & 240 & 240\end{array}$

$\begin{array}{lllllllllll}\text { N.H. } & 4 & 6 & 9 & 12 & 1415 & (65) & 14(6) & 14(6) & 14(6) & 13(6)\end{array}$

$0 \quad$ Número de hojas senescentes

Cuadro 2

ALTURA DE PLANTA (AP) Y NÚMERO DE HOJAS (NH) EN MANÍ

CARACT. DIAS DESPUES DE LA SIEMBRA

$\begin{array}{lllllllll}\text { EVALUAD. } 10 & 20 & 30 & 40 & 50 & 60 & 70 & 80 & 90\end{array}$

$\begin{array}{llllllllll}\text { AP. } & 11 & 20 & 28 & 40 & 55 & 62 & 60 & 88 & 94\end{array}$

$\begin{array}{llllllllll}\text { N.H. } & 4 & 12 & 25 & 39 & 53 & 66 & 75 & 85 & 94\end{array}$ 


\section{Cuadro 3}

Promedio de Peso Seco por Planta y Otros Órganos en Gramos (g) de Maíz

\begin{tabular}{lllllllllll}
\hline $\begin{array}{l}\text { Peso seco } \\
\text { (g) }\end{array}$ & 10 & 20 & 30 & 40 & 50 & 60 & 70 & 80 & 90 & 100 \\
\hline P1.To (PT) & 0,11 & 4,58 & 22,68 & 100.57 & 213,66 & 177,94 & 206.81 & 217,90 & 45,05 \\
& 78,19 & & & & & & & & \\
& & & & & & \\
Hojas (H) & & 1,59 & 9,57 & 24,79 & 56.78 & 39.20 & 42,71 & 48.30 & 7,85 & 28.44 \\
Tallos (T) & 0,11 & 2,99 & 13,11 & 75,78 & 141.51 & 99,67 & 91.99 & 123,80 & 13,40 & $21 \varnothing 3$ \\
Fl y Frt (FF) & & & & 15,37 & 39.07 & 72,11 & 45,80 & 23,80 & 28,35 \\
\hline
\end{tabular}

\section{Cuadro 4}

PROMEDIO DE PESO SECO POR PLANTA Y OTROS ORGANOS EN GRAMOS (G) DE MANI

\begin{tabular}{|c|c|c|c|c|c|c|c|c|c|}
\hline PS. SECO & & & DIAS D & ESPUES & E LA S & EBRA & & & \\
\hline (g) & 10 & 20 & 30 & 40 & 50 & 60 & 70 & 80 & 90 \\
\hline $\begin{array}{r}\text { PI.To (PT) } \\
53\end{array}$ & $\begin{array}{l}0,32 \\
50\end{array}$ & 1,31 & 6,52 & 12,76 & 21,86 & 32,07 & 43, & & 52,80 \\
\hline Hojas (H) & 0,17 & 0,80 & 3,72 & 7,00 & 9,02 & 10,07 & 13,55 & 12,20 & 13,64 \\
\hline Tallos (1) & & 0,15 & 0,51 & 2,75 & 5,48 & 7,05 & 9,35 & 13,75 & 12,20 \\
\hline & & & & & & & & & \\
\hline $\begin{array}{l}\text { Fly Frt }(\mathrm{FF}) \\
\mathrm{N}^{0} \text { Flor }(\mathrm{N})\end{array}$ & & & 0,05 & 0,28 & 5,79 & 12,65 & 15 , & 526,10 & 23,71 \\
\hline $\begin{array}{l}\mathrm{N}^{0} \text { Flor }(\mathrm{N} \\
\mathrm{N}^{0} \text { FRt }\left(\mathrm{N}^{0}\right.\end{array}$ & & & 13 & $\begin{array}{c}11 \\
3\end{array}$ & 7 & $\begin{array}{r}4 \\
22\end{array}$ & $\begin{array}{r}4 \\
22\end{array}$ & $\begin{array}{r}2 \\
23\end{array}$ & 1 \\
\hline
\end{tabular}

* Promedio peso seco de una semilla $0.41 \mathrm{~g}$. 


\section{Cuadro 5}

Índices de crecimiento en maíz

\begin{tabular}{|c|c|c|c|c|c|c|c|c|c|c|}
\hline \multicolumn{3}{|c|}{ IND. CRC. } & \multicolumn{6}{|c|}{ DIAS DESPUES DE LA SIEMBRA } & \multirow[b]{2}{*}{90} & \multirow[b]{2}{*}{100} \\
\hline & 10 & 20 & 30 & 40 & 50 & 60 & 70 & 80 & & \\
\hline $\mathrm{AF}$ & 0.34 & 5.90 & 36.92 & 71.14 & 169.67 & 100.60 & 99.86 & 94.60 & 19.50 & 23.10 \\
\hline IAF & 0.02 & 0.33 & 2.05 & 4.40 & 9.43 & 5.59 & 5.55 & 5.26 & 1.08 & 1.28 \\
\hline ICA & 0.14 & 3.13 & 12.66 & 54.47 & 79.08 & 24.98 & 20.18 & 7.76 & 70.00 & 23.17 \\
\hline ICC & 0.78 & 17.39 & 70.33 & 302.61 & 439.33 & 138.78 & 112.21 & 43.11 & 388.89 & 128.72 \\
\hline ICR & 0.73 & 0.48 & 1.12 & 1.04 & 0.52 & 0.12 & 0.10 & 0.04 & 1.10 & 0.38 \\
\hline IAN & 234.00 & 160.00 & 74.80 & 98.00 & 18.74 & 18.74 & 13.52 & 8.70 & 147.00 & 109.40 \\
\hline CAF & 310.00 & 129.00 & 163.0 & 79.00 & 57.00 & 57.00 & 48.00 & 43.00 & 43.00 & 30.00 \\
\hline DAF & 0.002 & 0.04 & 0.31 & 0.83 & 1.93 & 1.93 & 1.43 & 1.39 & 0.82 & 0.30 \\
\hline
\end{tabular}

\section{Cuadro 6}

\section{INDICES DE CRECIMIENTO EN MANÍ}

\begin{tabular}{lcrrrrrrrr}
\hline IND. & \multicolumn{10}{c}{ DIAS DESPUES DE LA SIEMBRA } \\
CREC. & 10 & \multicolumn{1}{c}{20} & \multicolumn{1}{c}{30} & \multicolumn{1}{c}{40} & \multicolumn{1}{c}{50} & \multicolumn{1}{c}{60} & \multicolumn{1}{c}{70} & \multicolumn{1}{c}{80} & \multicolumn{1}{c}{90} \\
\hline & & & & & & & & & \\
A & 0,43 & 2,59 & 9,81 & 15,15 & 19,59 & 24,50 & 26,92 & 31,11 & 26,67 \\
IAF & 0,05 & 0,32 & 1,23 & 1,90 & 2,45 & 3,06 & 3,37 & 4,01 & 3,71 \\
ICA & 0,06 & 0,69 & 3,64 & 4,36 & 6,36 & 7,14 & 7,68 & 6,82 & 0,49 \\
ICC & 0,75 & 8,63 & 45,50 & 54,50 & 79,50 & 89,25 & 96,00 & 85,25 & 6,13 \\
ICR & 0,17 & 0,99 & 1,12 & 0,48 & 0,37 & 0,27 & 0,20 & 0,15 & 0,01 \\
IAN & 76,00 & 57,34 & 30,13 & 2,28 & 37,14 & 32,63 & 29,83 & 23,05 & 1,59 \\
CAF & 134,00 & 198,00 & 150,00 & 119,00 & 90,00 & 76,00 & 63,00 & 61,00 & 55,00 \\
DAF & 0,003 & 0,02 & 0,09 & 0,18 & 0,25 & 0,32 & 0,37 & 0,42 & 0,44 \\
\hline
\end{tabular}

$\begin{array}{lll}\mathrm{AF} & = & \text { Area foliar }\left(\mathrm{dm}^{2}\right) \\ \mathrm{IAF} & = & \text { Indice de área foliar } \\ \mathrm{ICA} & = & \text { Indice de crecimiento absoluto }\left(\mathrm{g} \cdot \mathrm{sem}^{-1}\right) \\ \mathrm{ICC} & = & \text { Indice de crecimiento del cultivo }\left(\mathrm{g} \cdot \mathrm{m}^{-2} \mathrm{de} \text { suelo. sem } \mathrm{sem}^{-1}\right) \\ \mathrm{ICR} & = & \text { Indice de crecimiento relativo }\left(\mathrm{g} \cdot \mathrm{g}^{-1} \cdot \mathrm{sem}^{-1}\right) \\ \mathrm{IAN} & = & \text { Indice de asimilación neta }\left(\mathrm{g} \cdot \mathrm{m}^{-2} \mathrm{de} \mathrm{hoja.} \mathrm{sem}^{-1}\right) \\ \mathrm{CAF} & = & \text { Cociente de área foliar }\left(\mathrm{cm}^{2} \mathrm{de} \mathrm{hoja} \mathrm{g}^{-1}\right) \\ \text { DAF } & = & \text { Duración del área foliar }\left(\mathrm{m}^{2} \text { de hoja. } \mathrm{g}^{-1}\right)\end{array}$




\section{CONCLUSIONES}

- La curva típica de crecimiento "sigmoide" para altura de planta, se observó en el cultivo del maíz, mas no en el maní. El máximo número de hojas en maíz llegó a quince (15) a los 60 días, para descender hasta la cosecha; en maní el incremento del número de hojas fue continuo desde la siembra hasta la cosecha.

- En maíz los mayores valores del peso seco por planta encontrados se dieron a los 50 y 80 días; en cambio para maní la curva fue ascendente, teniendo valores más altos a los 80 y 90 días. En maní el número de flores es inversamente proporcional al número de frutos.

- Los mayores valores del AF en maíz fueron a los 50 y 60 días, siendo ellos de 169,67 y $100,60 \mathrm{dm}^{2}$; para maní, a los 80 y 90 días, fueron de 32,11 y 29,67 $\mathrm{dm}^{2}$.

- $\quad$ Los valores óptimos del IAF estuvieron comprendidos en maíz desde 4,40 hasta 9,43 y, para maní desde 3,06 hasta 4,01 .

- Las tasas más altas del ICA, ICC, ICR e IAN de maíz fueron 79,08 g.sem ${ }^{-1}$; $439,33 \mathrm{~g} \cdot \mathrm{m}^{-2} \cdot \mathrm{sem}^{-1} ; \quad 1,12 \mathrm{~g} \cdot \mathrm{g}^{-1} \cdot \mathrm{sem}^{-1}$ y $234,00 \mathrm{~g} \cdot \mathrm{m}^{-2}$ de hoja.sem ${ }^{-1}$, respectivamente; para maní se encontraron 7,68 g. $\mathrm{sem}^{-1}, 96,00 \mathrm{~g} \cdot \mathrm{m}^{-2} \cdot \mathrm{sem}^{-1} ; 1,12$ g. $\mathrm{g}^{-1} \cdot \mathrm{sem}^{-1}$ y $76,00 \mathrm{~g} \cdot \mathrm{m}^{-2}$ de hoja.sem ${ }^{-1}$.

- En maíz los índices estimados de CAF y DAF estuvieron comprendidos entre 310 a $30 \mathrm{~cm}^{2}$ de hoja. $\mathrm{g}^{-1}$ y de 0,002 a $1,93 \mathrm{~m}^{2}$ de hoja.sem ${ }^{-1}$. Pero en maní estas tasas fueron de 198 a $55 \mathrm{~cm}^{2}$ de hoja.g $\mathrm{g}^{-1}$ y de 0,003 a $0,44 \mathrm{~m}^{2}$ de hoja.g $\mathrm{g}^{-1}$, respectivamente.

- En los cultivos de maíz y maní las eficiencias fotosintéticas medias fue de $1,27 \%$ y $0,75 \%$; los índices de cosechas (IC) de $36 \%$ y $25 \%$ y, las eficiencias foliares (Ef) medias de 0,59 y 0,67 g.dm ${ }^{-2}$ de hoja, respectivamente.

\section{BIBLIOGRAFIA}

BENITES, J. (1988). "Estimación de la radiación solar en Iquitos". en Folia Amazónica. IIAP-Iquitos, Perú. Vol. 1(1)1 13-128. 149p.

EVANS, L. (1983). Fisiología de los cultivos. Trad. H. Gonzales. Primera Edic. Español - Argentina Editorial Hemisferio Sur. 400 p.

LUCCHESI, A. (1987). "Factores de la producción vegetal", en Ecofisiologia de la Producción Agrícola. Asociación Brasilera para Investigación del Fósforo y Potasio. Paracicaba-SP-Brasil. 1-1-. 250 
MACHADO, E. (1985). "Eficiencia fotosintética". Seminario de Biotecnología Agrícola. Paracicaba-Brasil. FEALQ. 175-200 p.

MEDINA, E. (1977). Introducción a la Ecofisiología vegetal. Instituto Venezolano de Invest. Cientif. OEA. Caracas-Venezuela. 102 p.

SANCHEZ, H. (1973). "V. Reunión de Maiceros de la Zona Andina. CochabambaBolivia”. del 26 al 30 marzo. 30p.

SOPLIN, J. (1991). "Efecto del follage en el crecimiento y producción del Caupí (Vigna sinensis L.) V.'San Roque' Bajo dos Densidades de Plantas”, en Revista Conocimiento-UNAP, Iquitos, Perú. Vol. 2 (1):73-87. 161 p.

YOSHIDA, 5. (1972). "Physiological Aspect of Grain Yield", en Annual Review of Plant Physiology. Palo Alto. 3 (23):67-437 p. 


\section{Grafico 1}

MAIZ: CURVAS DE CRECIMIENTO PARA:

ALTURA DE PLANTA (AP)

NUMERO DE HOJAS POR PLANTA (NH)

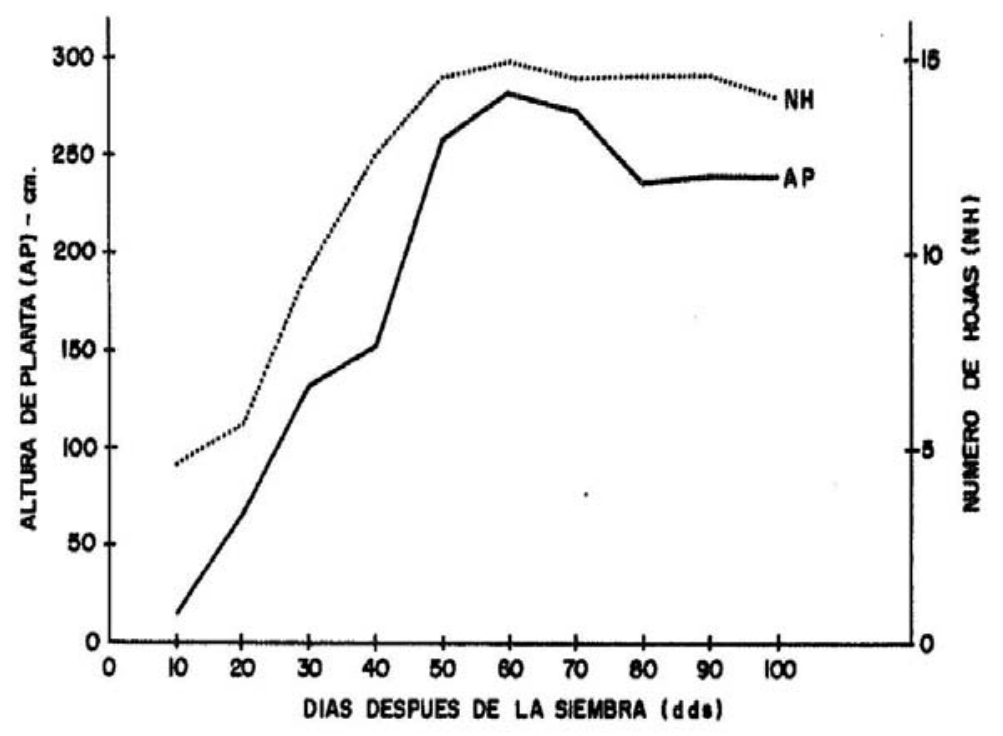




\section{Grafico 2}

MANI: CURVAS DE CRECIMENTO PARA:

ALTURA DE PLANTAS (AP)

NUMERO DE HOJAS POR PLANTA (NH)

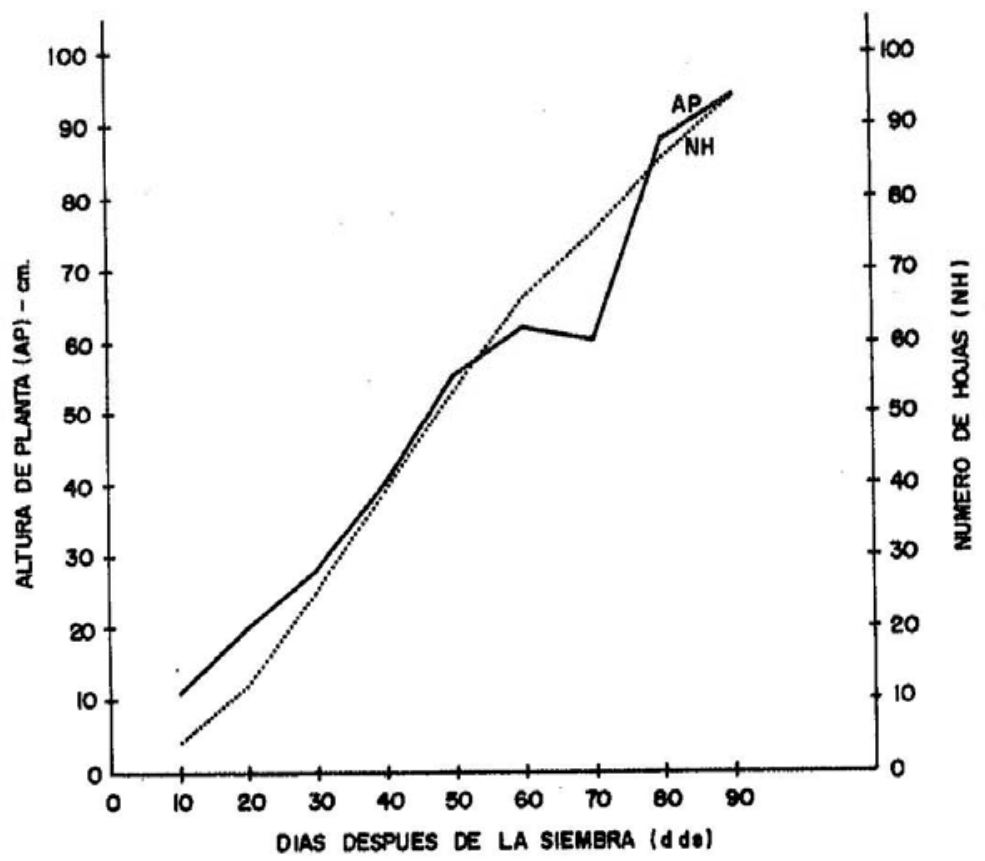




\section{Grafico 3}

MAIZ: CURVAS DE INCREMENTO DE PESO POR PLANTA Y OTROS ORGANOS EN GRAMOS (g)
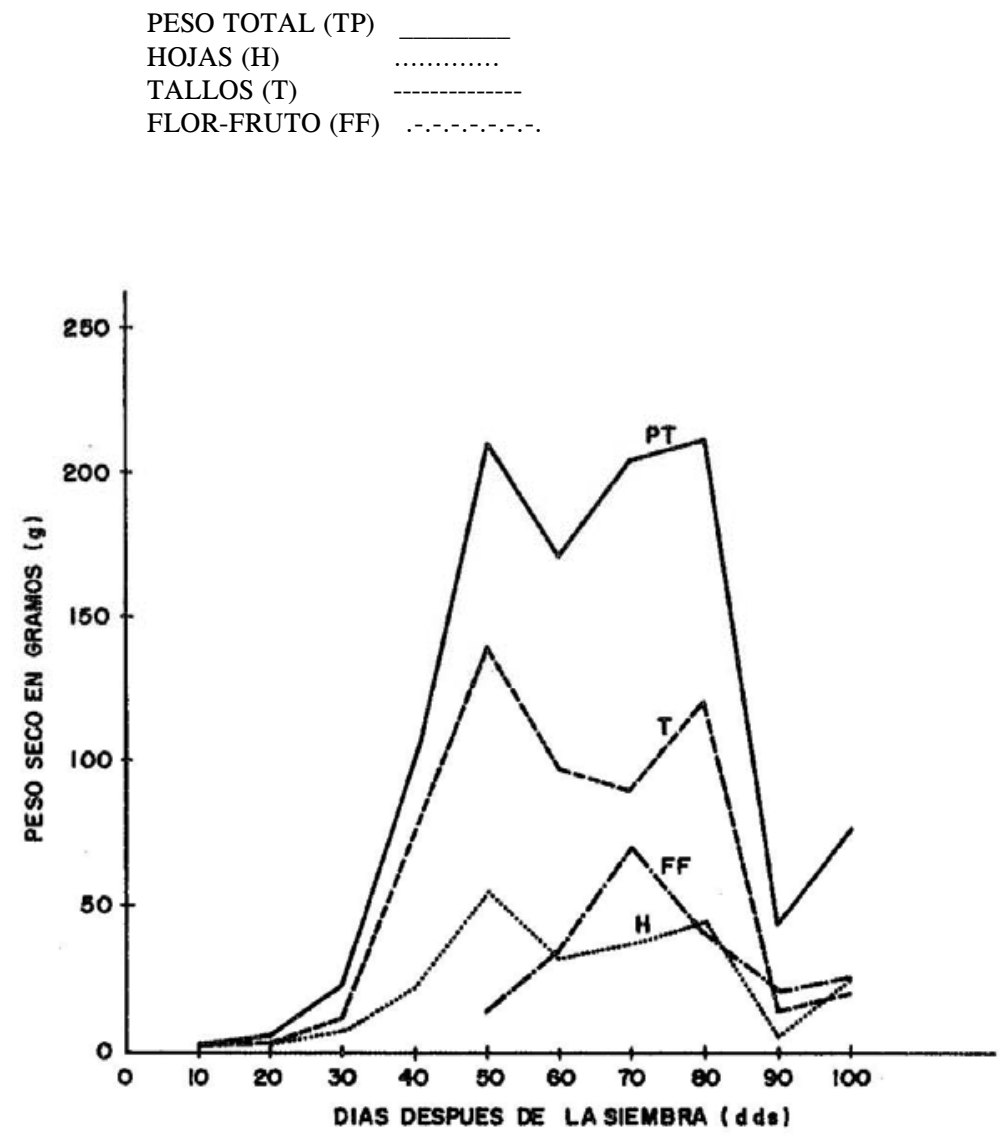


\section{Grafico 4}

MANI: CURVAS DE INCREMENTO DE PESO POR PLANTA Y OTROS ORGANOS EN GRAMOS (g)
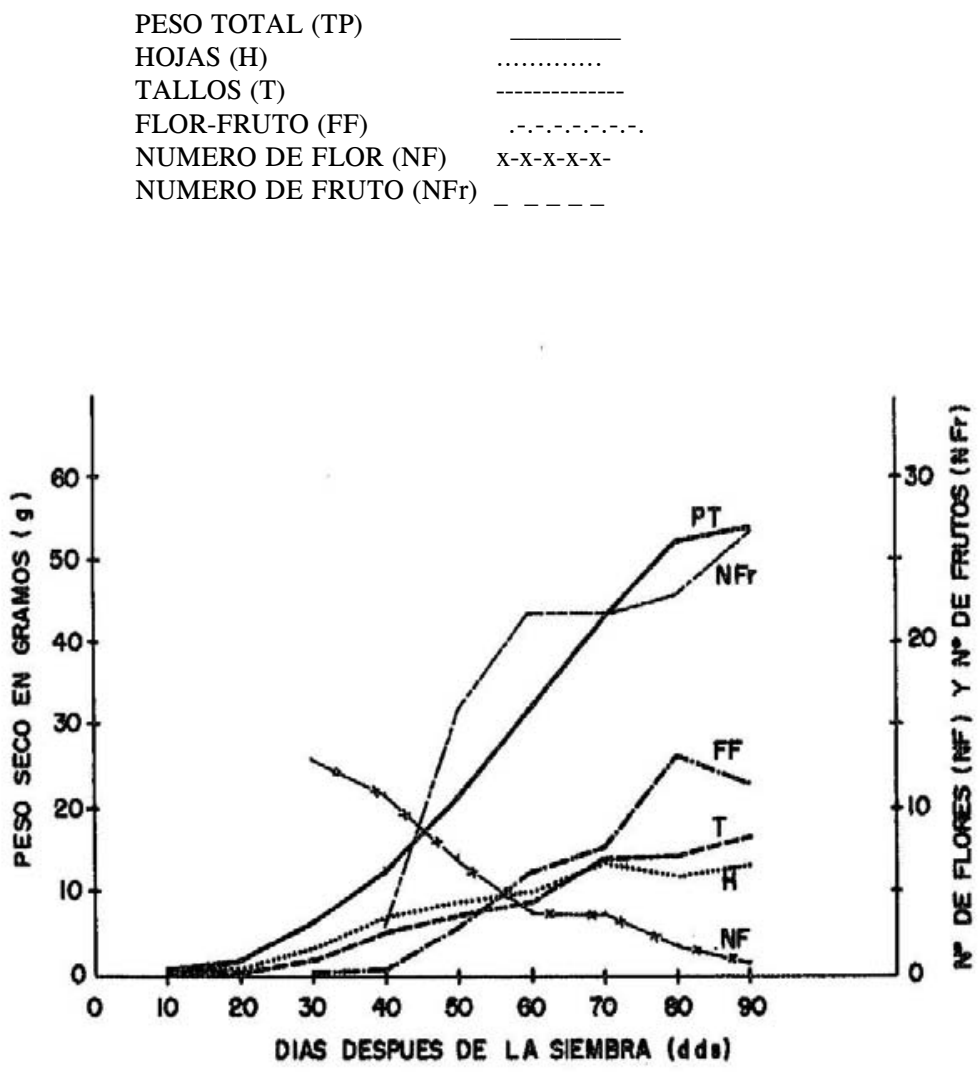


\section{Grafico 5}

MAIZ: CURVA DE:

AREA FOLIAR (AF) -dm ${ }^{2}$

INDICE AREA FOLIAR (IAF)

INDICE CRECIMIENTO ABSOLUTO (ICA)-g.gem ${ }^{-1}$

INDICE CRECIMIENTO ABSOLUTO (ICA)-g. $\mathrm{m}^{-2} \cdot \mathrm{sem}^{-1}$

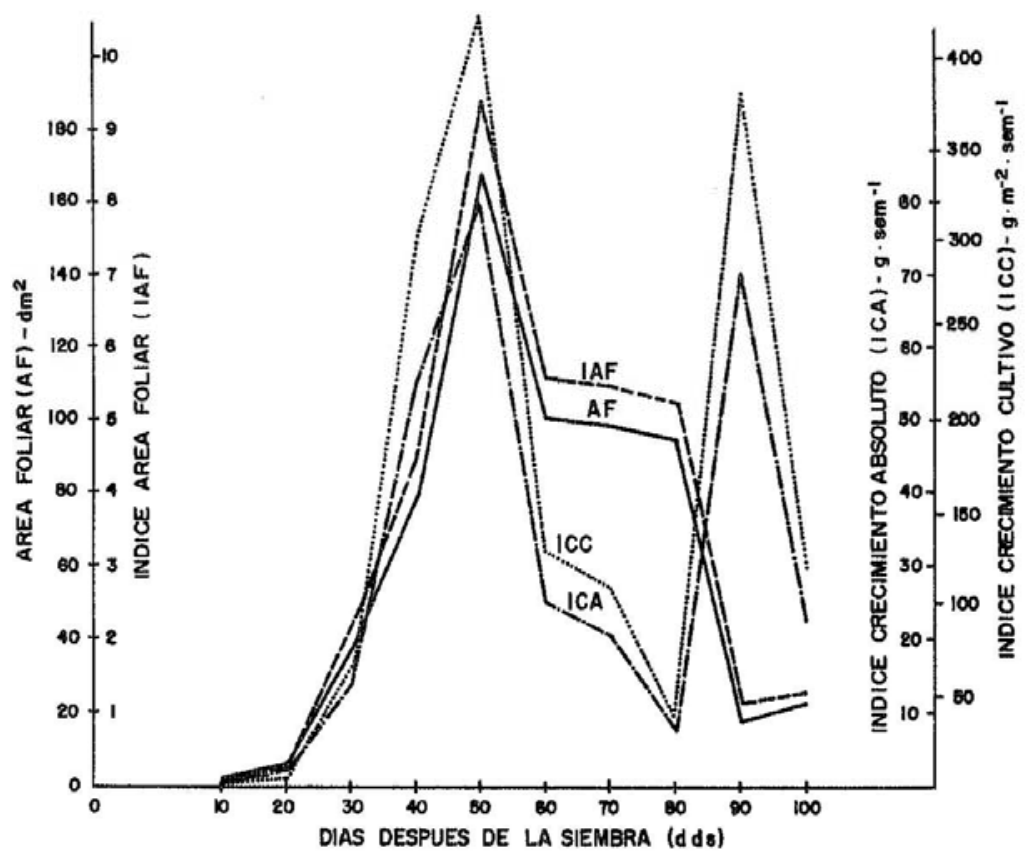




\section{Grafico 6}

MANI: CURVA DE:

AREA FOLIAR (AF) - $\mathrm{dm}^{2}$

INDICE AREA FOLIAR (IAF)

INDICE CRECIMIENTO ABSOLUTO (ICA)-g. gem $^{-1}$

INDICE CRECIMIENTO ABSOLUTO (ICA)-g. $\mathrm{m}^{-2} \cdot \mathrm{sem}^{-1}$

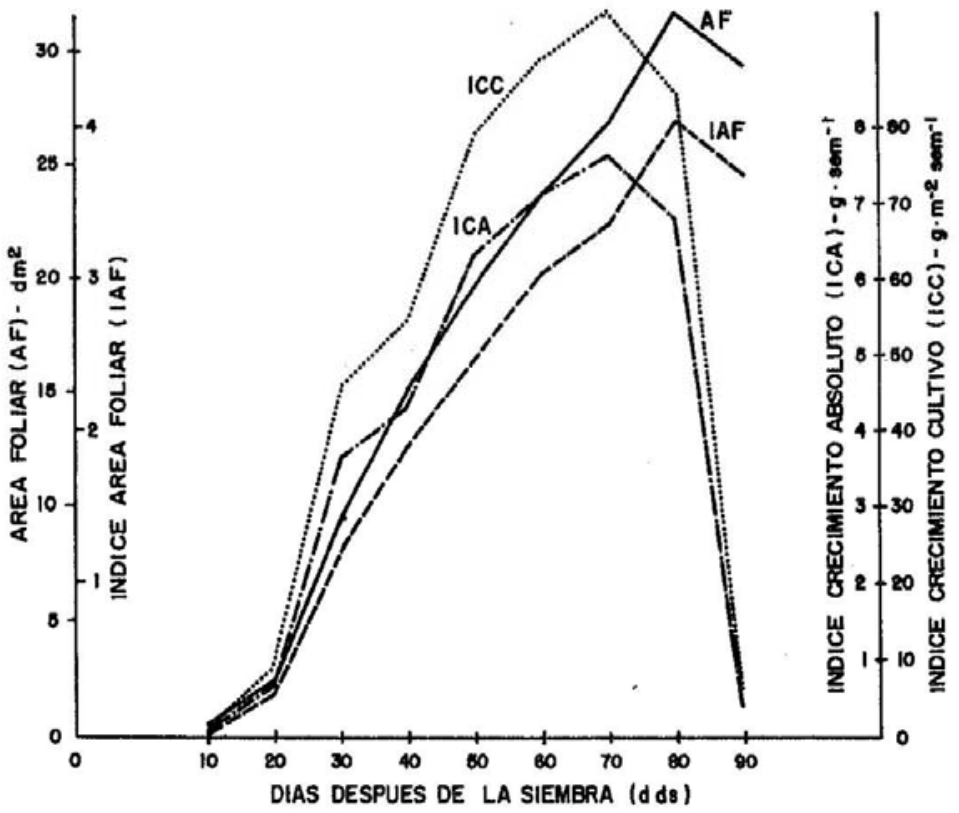




\section{Grafico 7}

MAIZ: CURVA DE:

INDICE CRECIMIENTO RELATIVO (ICR)-g. $\mathrm{g}^{-1} \cdot \mathrm{Sem}^{-1}$

INDICE ASIMILACION NETA (IAN) $-\mathrm{g} \cdot \mathrm{m}^{-2} \cdot \mathrm{sem}^{-1}$ COCIENTE AREA FOLIAR (CAF) $-\mathrm{cm}^{2} \mathrm{~g}^{-1}$

DURACION AREA FOLIAR (DAF)- $\mathrm{m}^{-2} \mathrm{~g}^{-1}$

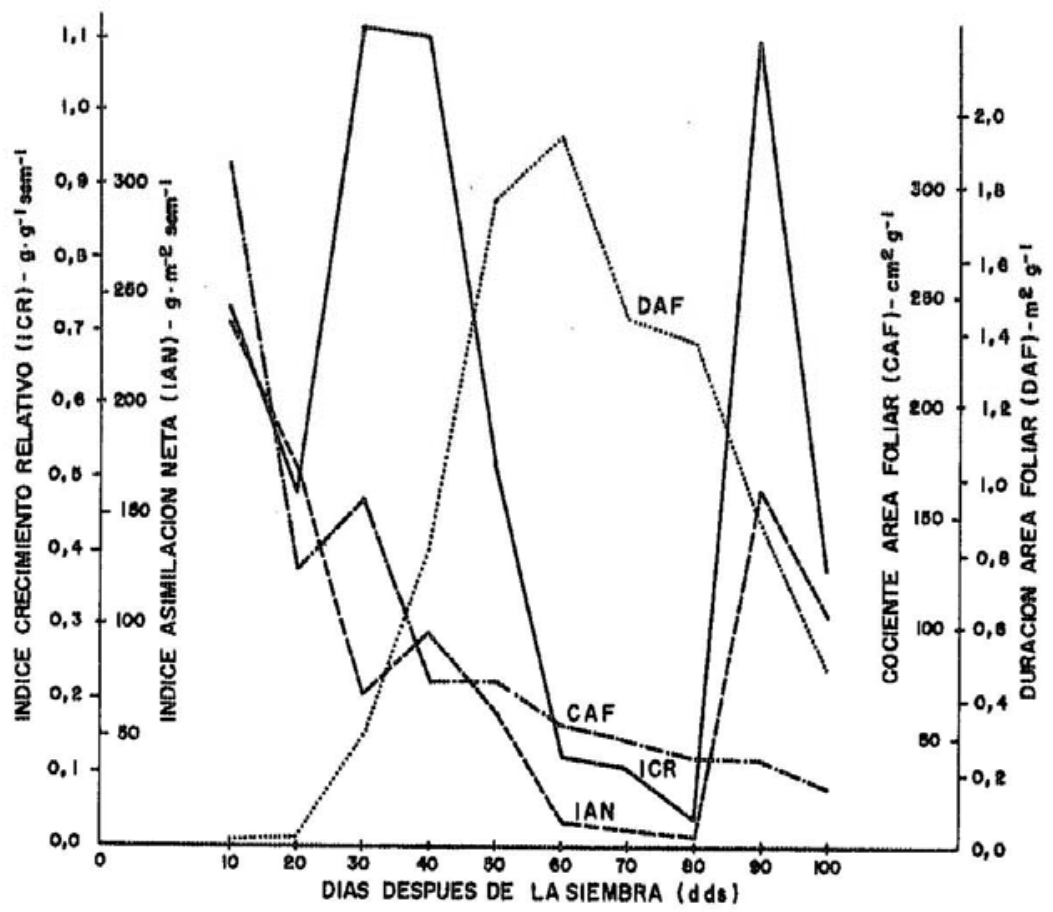




\section{Grafico 8}

\section{MANI: CURVA DE:}

INDICE CRECIMIENTO RELATIVO (ICR)-g. $\mathrm{g}^{-1} \cdot \mathrm{Sem}^{-1}$

INDICE ASIMILACION NETA (IAN) $-\mathrm{g} \cdot \mathrm{m}^{-2} \cdot \mathrm{sem}^{-1}$

COCIENTE AREA FOLIAR (CAF)- $\mathrm{cm}^{2} \mathrm{~g}^{-1}$

DURACION AREA FOLIAR (DAF)- $\mathrm{m}^{-2} \mathrm{~g}^{-1}$

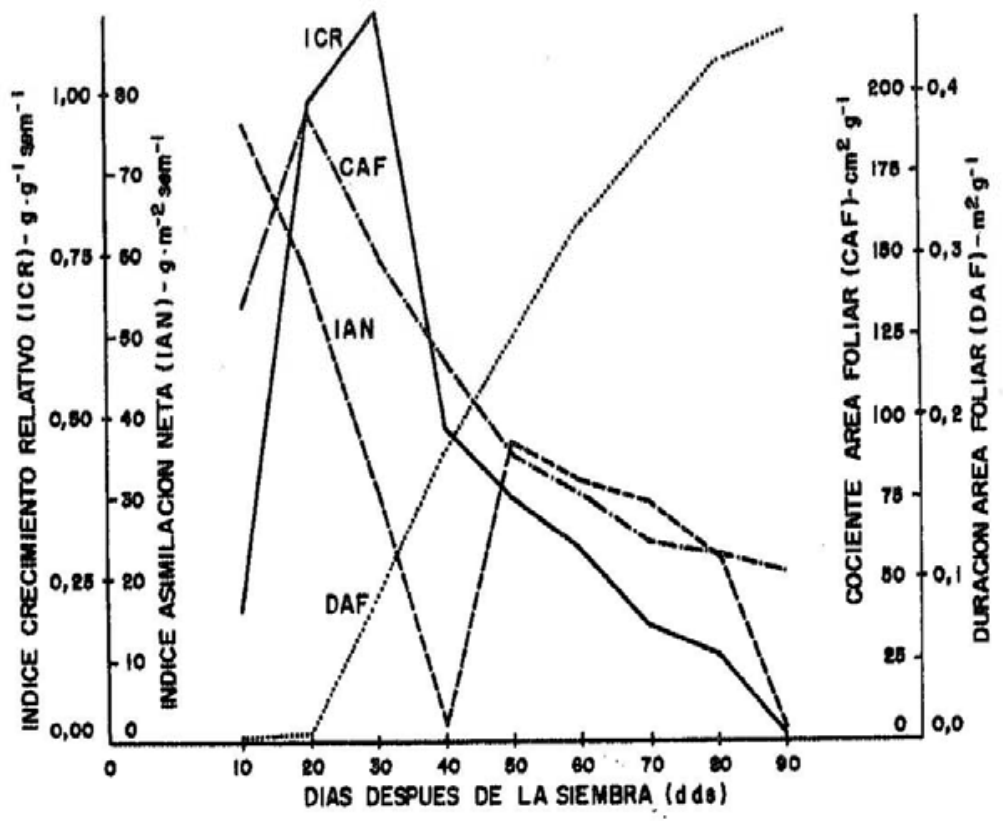

